\title{
Frontonasal encephalocele with subependymal heterotopias and cerebellar dysplasia: a rare case report with review of literature
}

\author{
Kunwar Pal Singh ${ }^{1,2^{*}}$ (D) Sukhdeep Kaur ${ }^{1}$ and Bikramjit Singh Sidhu ${ }^{1}$
}

\begin{abstract}
Background: Encephaloceles are herniation of brain parenchyma through the defect in the dura and the skull bones. This case report reveals a rare association of frontonasal encephalocele, subependymal nodular heterotopias and cerebellar dysplasia with review of literature.

Case presentation: Frontonasal encephalocele is a rare entity. We report an 18-months-old male child who presented to the department of pediatrics with chief complaint of congenital swelling in the region of forehead. Swelling was increasing in size as told by the parents of the child. The child was born full term with normal vaginal delivery. There was no history of any obstetrical complications. The swelling was soft, transilluminant and pulsatile in nature. He was referred to the department of radio diagnosis for imaging evaluation. His CT and MRI images revealed defect in the anterior cranial fossa with herniation of meninges and brain parenchyma through it. There was also evidence of subependymal nodular heterotopias in this patient along with hypoplasia of left cerebellar hemisphere and left middle cerebellar peduncle with left cerebellar dysplasia.
\end{abstract}

Conclusions: This case report describes the coexistence of frontonasal encephaloceles, subependymal nodular heterotopias and cerebellar dysplasia, which is a very rare association.

Keywords: Case report, Encephalocele, Frontonasal, Heterotopia, Magnetic resonance imaging

\section{Background}

Encephalocele is part of spectrum of neural tube defect. The incidence of encephalocele is not uncommon in the developing countries. It varies from 1 to 3 per 1000 live births across the world. Various neural tube defects include anencephaly, encephalocele and spina bifida. These are common congenital malformations and have with multifactorial pathogenesis. Their prevalence varies in different populations [1]. Anterior encephalocele is a rare entity among its other types. Its incidence in

\footnotetext{
*Correspondence: kpsdhami@gmail.com

${ }^{1}$ Sri Guru Ram Das Institute of Medical Sciences and Research, Amritsar,

Punjab, India

Full list of author information is available at the end of the article
}

the western world ranges from $1 / 35,000$ to $1 / 40,000$ live births, however, in Asian continent, it increases to about $1 / 5000$ live births. Crania bifida is a term which includes encephaloceles and meningoceles. Although these are less common as compared to spina bifida, bifida, these are not uncommon. Also, clinical management of these conditions can be difficult [2]. Occiput is the most common site for encephaloceles as far as the literature is concerned. These occur commonly in the mid-sagittal plane anteriorly from nasofrontal region and posteriorly to the occipital region. Occipital encephalocele is common in western world, and the anterior encephaloceles are more often seen in Asian countries. Anterior encephalocele is a less commonly reported central nervous system 
malformation and it has more predilection for Southeast Asian region. Nasal encephaloceles are part of anterior defect which can be further divided into frontoethmoidal and basal varieties. Both these conditions are, but frontoethmoidal variety has a relatively higher incidence (1:5000) in Southeast Asian territory. The pathogenesis of encephaloceles is explained as disturbance in the separation of surface later of the ectoderm (epithelial layer) and the neurectoderm (nervous tissue) in the midline just after closure of the neural folds. So it is better to call it as a late neurulation defect occurring during the 4th week of gestation. This disturbed separation of layers is related to cell death or apoptosis. Frontoethmoidal encephaloceles present as a soft facial mass covered with normal intact skin, whereas basal variety may cause nasal stuffiness or obstruction. CT or MR imaging helps in delineating the nature of the herniated structures well $[3,4]$.

\section{Case presentation}

An 18-months-old male child presented to the department of pediatrics with chief complaint of congenital swelling in the region of forehead and hypertelorism. Swelling was increasing in size as told by the parents of the child. The baby was born at full-term normal vaginal delivery without any complications. The swelling was soft, transilluminant and pulsatile in nature. He was referred to the department of radio diagnosis for imaging evaluation. MRI of patient was done using TSE T2W and TSE T1W sequences in axial plane, TSE T2W sequence in coronal and saggital planes. MRI finding revealed a large defect in the frontonasal region and herniation of brain parenchyma through the defect into a sac-like structure. The defect was midline in position. There was also evidence of ectopic gray matter tissue along the ventricular walls bilaterally suggestive of subependymal heterotopia. There was hypoplasia of left cerebellar hemisphere and thinning of left middle cerebellar peduncle. An oval-shaped soft tissue was observed near the left cerebellar hemisphere medially along the lateral wall of fourth ventricle and projecting into its lumen. Its signal intensity was isointense to gray matter on T1W and T2W images (Fig. 1). It was labeled as either heterotopic gray matter tissue or dysplastic cerebellar parenchyma. CT scan of base of skull was performed in axial plane with reconstruction in the coronal and saggital planes. Soft tissue window revealed the herniated
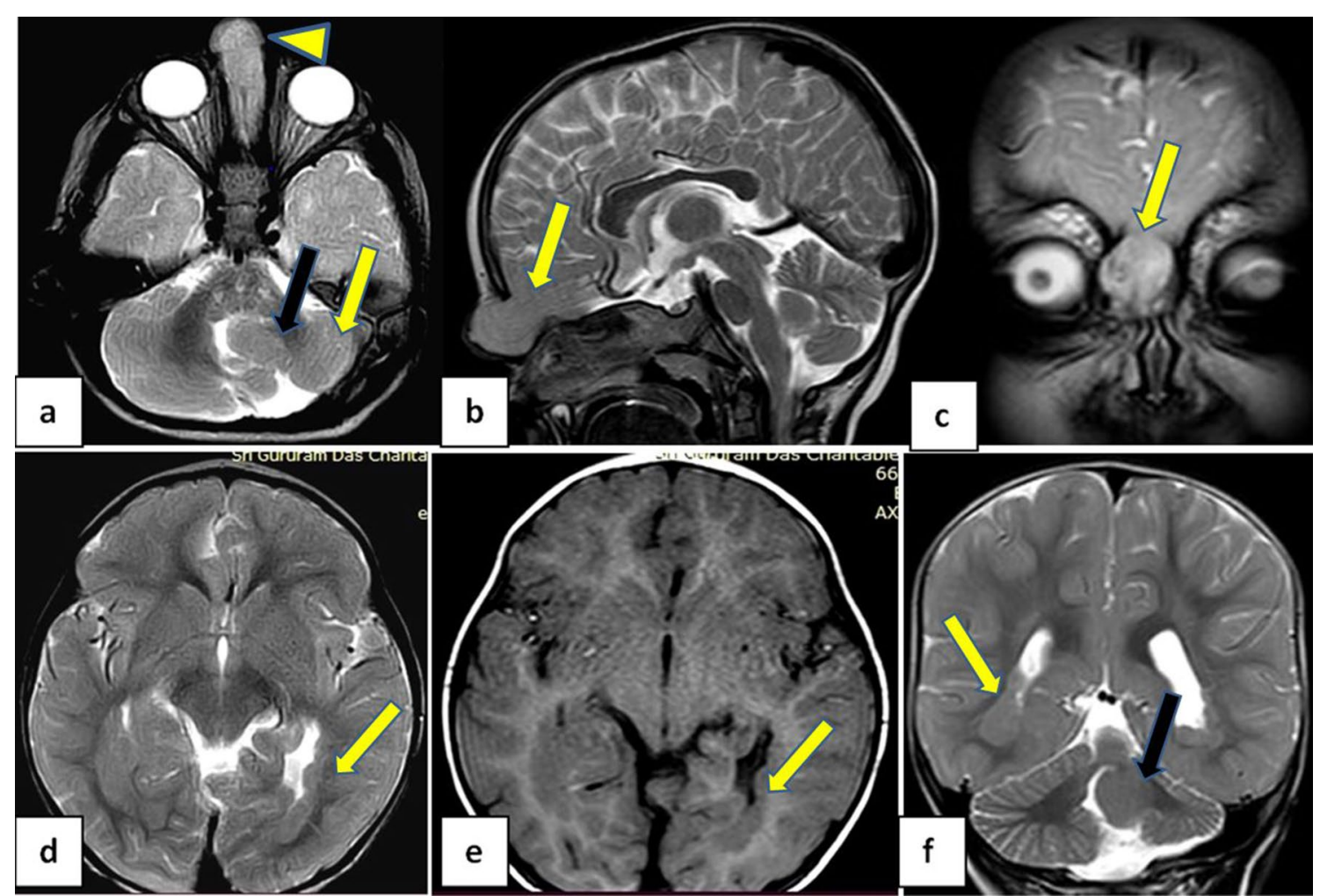

Fig. 1 TSE T2W images in axial (a), saggital (b) and coronal (c) planes show herniation of frontal lobe parenchyma through the defect (yellow arrow head in a and yellow arrows in $\mathbf{b}$ and $\mathbf{c}$ ). There is cerebellar hypoplasia and heterotopia/dysplastic cerebellar tissue on the left side (yellow and black arrows in a). TSE T2W (d), SE T1W (e) images in axial plane and TSE T2W image in coronal plane (f) show subependymal heterotopias bilaterally; marked as yellow arrows. Black arrow in figure f shows cerebellar hypoplasia and heterotopia/dysplastic cerebellar tissue 
cerebral parenchyma in nasofrontal region (Fig. 2). Bony algorithm in axial plane along with three-dimensional volume rendering technique (VRT) was used to measure the size of the defect in the three dimensions. Frontal and basal VRT views of skull were interpreted for the dimensions. The defect measured $1.42 \times 1.14 \times 1.08 \mathrm{~cm}$ in size (Fig. 2). Final imaging diagnosis of frontonasal encephalocele with subependymal heterotopias and cerebellar dysplasia was made.

\section{Discussion}

Frontal encephaloceles are a rare entity [2]. A retrospective study on neural tube defects was done by Ghosh Soumyodhriti et al. According to this study, the incidence of encephalocele was $14 \%$, however, they did not discussed about the defects in the frontal bone in their study. The probable reason for this was the absence of cases in their study [5]. The basic etiology of encephalocele is the defect in the ectodermal layer which happens in utero. The cells of ectodermal layer which form the skull do not cover over the brain parenchyma. This effect causes outward protrusion of brain parenchyma and meninges through these osseous defects. Encephalocele may be present anywhere in head but most commonly located in midline location in the region of occiput [6]. The etiology is multifactorial. Few studies reveal that folic acid supplementation dramatically reduces the occurrence of these neural tube defects. Its use is advised at least 4 weeks before conception and is further continued till the 13 week of pregnancy [7].

Clinical presentations in these patients are very variable. Most of them presented with soft tissue swelling in the head. Most of the frontoethmoidal encephaloceles clinically presented with lump at the level of nasal bridge. Few of the patients may have facial dysmorphism such as proptosis and hypertelorism. Basal encephalocele is usually present with swelling in the region of nasopharynx and clinical symptoms of airway obstruction, nasal bleeding and CSF rhinorrhoea. These patients can also present epilepsy, delayed milestones, visual disturbances, gait abnormalities, microcephaly, paraplegia and intracranial hypertension [8-10].

As far as reporting format of encephalocele is concerned, familiarity with the anatomy is very important. The classification of encephaloceles based on their anatomical location (especially the anterior ones) has been
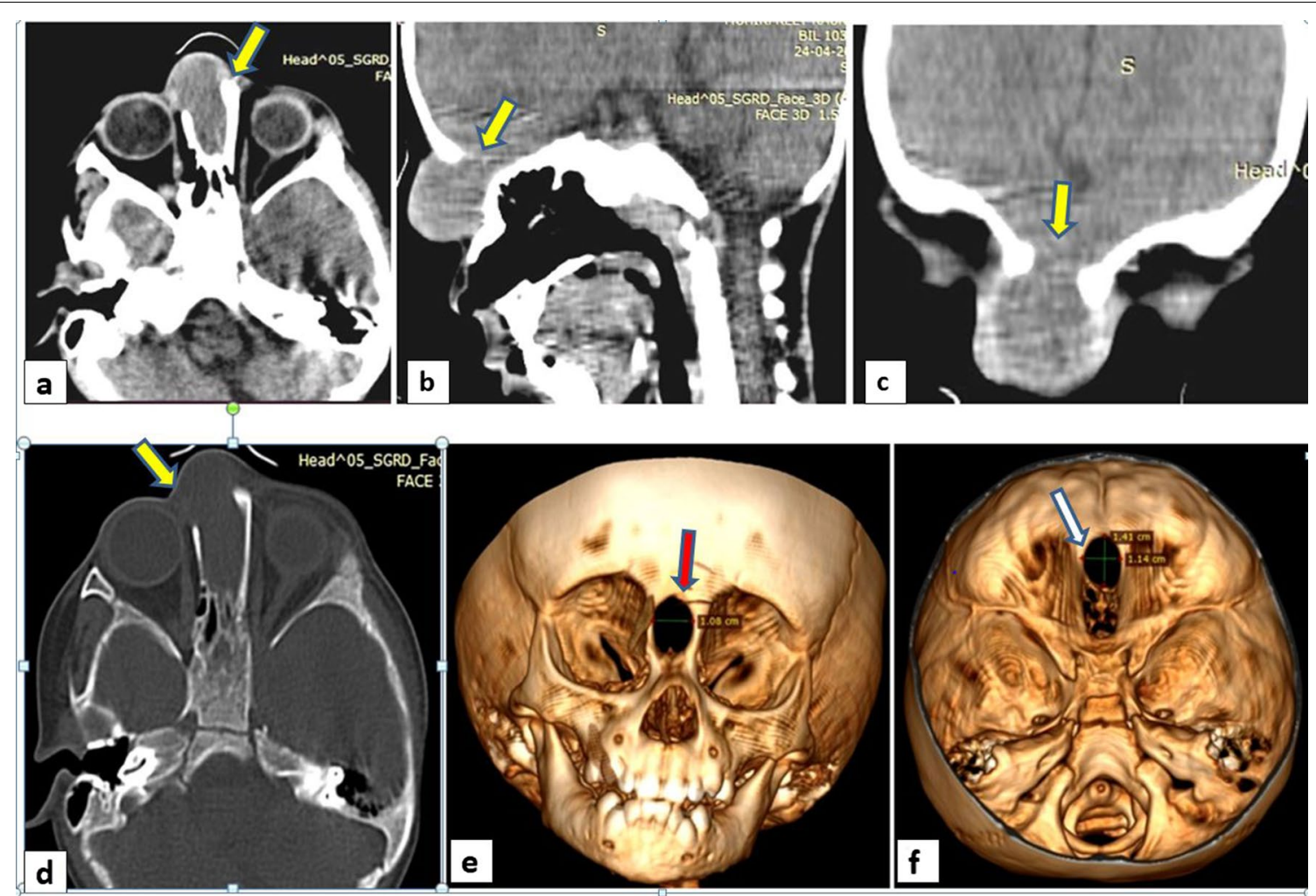

Fig. 2 Non-contrast CT base of skull in axial plane (a) with saggital (b) and coronal (c) reconstruction with soft tissue window settings shows herniation of frontal lobe parenchyma through the defect (yellow arrows). Non-contrast CT base of skull in axial plane with bony algorithm (d) shows the bony defect. Three-dimensional CT volume rendering technique (VRT) with frontal (e) and basal views (f) shows oval-shaped defect in the nasofrontal region with measurements (red and white arrows) 
well described in the studies. The encephaloceles in the nasofrontal region occur due to abnormal regression of the dural diverticulum from the prenasal space $[9,11]$. Magnetic resonance imaging helps in characterizing the anatomical site of the encephalocele, characterize the contents of the sac and also identify any other associated congenital malformations of the central nervous system. These imaging features further help in pre-surgical planning and prognosis of the disease. Computed tomography scanning can also show the osseous anatomy very well with special use of volume rendering technique (VRT) to see the three-dimensional view of the osseous defects. Routine MRI sequences include TSE T1W and TSE T2W sequences in axial, coronal and sagittal planes. Magnetic resonance venography and angiography may also be required to determine the relation of encephaloceles and their contents with cerebral venous sinuses and arteries. This is done to avoid any intraoperative vascular injury and to prevent any ischemic event post-operatively. Heavily weighted T2-weighted or CISS images should be done while evaluating the pathologies in the fronto-ethmoidal region, to delineate the tracts and various intracranial connections. These sequences can also help to differentiate nasal glioma, dorsal dermal sinus and encephalocele.

CT scan is highly useful in accurately assessing the dimensions of the skull vault defects, so that appropriate surgical planning can be done. As far as location is concerned, the anterior encephaloceles are located anterior to the bregma. They are subdivided into sincipital variety and basal variety depending upon their location in the region of the nasal bridge and the cribriform plate and sphenoid sinus, respectively $[9,10]$. Sincipital encephaloceles are further subdivided into two main types: frontoethmoidal and interfrontal.

Interfrontal variety is seen between bregma and the nasal bone and is often midline in location adjacent to the caudal aspect of the metopic suture. The frontoethmoidal variety occurs through the foramen cecum, that is located anterior to the crista galli. The frontoethmoidal encephaloceles are subcategorized into nasofrontal, nasoethmoidal and nasoorbital types [12].

Confirmative radiological diagnosis of frontal encephalocele is made with the help of CT and MRI of the brain, as both these imaging modalities have different roles. Associated craniofacial anomalies can also be visualized by combining these modalities.

As far as gray matter heterotopia is concerned, it is defined as a neuronal migration disorder in which the cortical neurons are present in an abnormal anatomical location. There is arrest in the radial migration of neurons from the germinal matrix within the wall of the ventricle to the developing cerebral cortex in the intrauterine life. This usually occurs between six to sixteen of intrauterine life. The disease is usually discovered during imaging evaluation of children with neurodevelopmental abnormalities, seizures or it can be incidental.

The exact etiopathogenesis of heterotopia is not well understood, but these pathologies can present with typical clinic-radiological syndromes. Types of heterotopia include nodular, laminar and leptomeningeal, (double cortex or band heterotopia) varieties, however, the classification has no significant clinical impact [13].

Neurophysicians have classified heterotopia into subependymal, subcortical and band varieties based on findings on magnetic resonance imaging findings. The three patterns were classified on the basis of the site and appearance of the ectopic gray matter. The subependymal variety has small foci of gray matter situated along the subependymal lining in close relation with the walls of the ventricles. Subcortical heterotopia is seen within subcortical and deep white matter. Band heterotopia was described as a symmetrical thick band of gray matter tissue with smooth margins that is seen with in the layers of the white matter $[13,14]$.

On MRI, the subependymal heterotopias appear as round to ovoid subependymal nodules, located just beneath and abutting the ependymal lining of the lateral ventricles. They may protrude slightly into the ventricular lumen causing irregular ventricular outline. The number and size of nodules may vary from small size to a sheet-like layer of conglomerate nodules of gray matter. Important point is that these nodules do not enhance after intravenous administration of gadolinium. This point distinguishes them from the subependymal nodules of tuberous sclerosis, as these nodules are not isointense to gray matter and they enhance after intravenous gadolinium administration.

Cortical heterotopias appear on MRI as areas of ectopic gray matter within the cerebral white matter. The size varies from a few centimeters to large focal mass-like lesions that may cause distortion of the adjacent ventricle. These also do not show enhancement on post-contrast sequences.

Band heterotopias appear as smooth, bilateral and symmetric ribbon type areas of ectopic gray matter in the central white matter between the cerebral cortex and the ventricular surface. These areas are separated from the cortex by normally myelinated cerebral white matter. Bands are not in continuation with the overlying cortex. They do not contain blood vessels or cerebrospinal fluid. The thicker the band; the worse is the clinical disability and also higher prevalence of developmental delay [14]. Only a few cases of frontonasal encephalocele with subependymal nodular heterotopias has been described in the literature [15]. Few cases are there in the literature 
that demonstrates the association of anterior encephalocele with subependymal nodular heterotopia, cortical dysplasia and epilepsy [16]. Roelens et al. evaluated the presence of nodular subependymal heterotopia in two case series with posterior encephalocele [17]. Lotfi presented the first case of periventricular heterotopia associated with cervical meningocele. Our case report further emphasizes the role of MRI brain in the evaluation of these congenital neural tube defects [18]. Various pediatric cerebellar malformations have been described in the literature which include cerebellar hypoplasia, aplasia, dysplasia and dandy walker malformations. Most of these patients are present with global develeopmental delay [19]. To the best of our knowledge, no association of unilateral cerebellar hypoplasia and dysplasia with anterior encephalocle and heterotopias has been described in the literature. We describe an unusual rare case of 18-months-old boy with a frontonasal soft tissue swelling with radiological evaluation showing frontonasal encephalocele, subependymal nodular heterotopias and left cerebellar atrophy with dysplasia. The limitation of the case report was that patient did not come for follow-up.

\section{Conclusions}

This case report describes the coexistence of frontonasal encephaloceles, subependymal nodular heterotopias and cerebellar dysplasia, which is a very rare association.

\section{Abbreviations}

MRI: Magnetic resonance imaging; CT: Computed tomography; VRT: Volume rendering technique; CSF: Cerebrospinal fluid.

\section{Acknowledgements}

None.

\section{Authors' contributions}

KS contributed to study conception and design, acquisition of data, analysis and interpretation of data, drafting of manuscript. SK contributed to drafting of manuscript and critical revision. BS contributed to drafting of manuscript. All authors have read and approved the manuscript and ensured that this is the case. All authors read and approved the final manuscript.

\section{Funding}

Not applicable.

\section{Availability of data and materials}

Available in the department.

\section{Declarations}

\section{Ethics approval and consent to participate}

Written informed consent was taken from parents of the child for imaging. Written informed consent was taken from patient's parents.

\section{Consent for publication}

Written informed consent was taken.

\section{Competing interests}

No competing interests.

\section{Author details}

${ }^{1}$ Sri Guru Ram Das Institute of Medical Sciences and Research, Amritsar, Punjab, India. ${ }^{2}$ Amritsar, India.

Received: 22 June 2021 Accepted: 9 August 2021

Published online: 16 August 2021

\section{References}

1. Rad IA, Farrokh-Islamlou HR, Khoshkalam M (2008) Neural tube defects prevalence in a hospital-based study in Urmia. Iran J Child Neurol 2(3):19-23

2. Barrow N, Simpson DA (1966) Cranium bifidum: investigation, prognosis and management. Aust Paediatr J 2:20-26

3. Mahapatra AK, Agrawal D (2006) Anterior encephaloceles: a series of 103 cases over 32 years. J Clin Neurosci 13:536-539

4. Hoving EW (2000) Nasal encephaloceles. Child's Nerv Syst 16:702-706

5. Soumyodhriti G, Mujaldevikram Singh S, Kumar G (2016) Neural tube defects-demography in a tertiary care center. IOSR J Dent Med Sci IOSRJDMS 1(15):80-82

6. Siffel C, Wong LY, Olney RS, Correa A (2003) Survival of infants diagnosed with encephalocele in Atlanta, 1979-98. Paediatr Perinat Epidemiol 17(1):40-48

7. MRC Vitamin Study Research Group (1991) Prevention of neural tube defects: results of the medical research council vitamin study. Lancet 338(60):131-137

8. Barkovich AJ (2005) Congenital anomalies of the spine. In: Barkovich AJ (ed) Pediatric neuroradiology, 4th edn. Lippincott Williams \& Wilkins, Philadelphia, pp 801-868

9. Bhagwati SN, Mahapatra AK (1999) Encephalocele and anomalies of the scalp. In: Choux M, Di Rocco C, Hockley AD, Walker JL (eds) Pediatric neurosurgery. Churchill Livingstone, London, pp 101-120

10. Lowe LH, Booth TN, Joglar JM, Rollins NK (2000) Midface anomalies in children. Radiographics 20(4):907-922

11. Barkovich AJ, Vandermarch P, Edwards MSB, Cogen PH (1991) Congenital nasal masses: CT and MR imaging features in 16 cases. AJNR Am J Neuroradiol 12(1):105-116

12. Pal NL, Juwarkar AS, Viswamitra S (2021) Encephalocele: know it to deal with it. Egypt J Radiol Nucl Med 52:105

13. Barkovich AJ (2000) Morphologic characteristics of subcortical heterotopia: MR imaging study. AJNR Am J Neuroradiol 21:290-295

14. Donkol RH, Moghazy KM, Abolenin A (2012) Assessment of gray matter heterotopia by magnetic resonance imaging. World J Radiol 4(3):90-96

15. Balaji R, Mangaleswaran B, John R (2010) Frontoethmoidal encephalocele with subependymal nodular heterotopia: an unusual association. A case report. Neuroradiol J 23(3):317-320

16. Rojas L, Melvin JJ, Faerber EN, Valencia I (2006) Anterior encephalocele associated with subependymal nodular heterotopia, cortical dysplasia and epilepsy: case report and review of the literature. Eur J Paediatr Neurol 10(5-6):227-229

17. Roelens FA, Barth PG, van der Harten JJ (1999) Subependymal nodular heterotopia in patients with encephalocele. Eur J Paediatr Neurol 3(2):59-63

18. Lotfi M, Iranpour P, As'adi K (2011) Cervical meningocele associated with subependymal nodular heterotopia. Clin Imaging 35(3):214-216

19. Ibrahim RSM, Hachem RH (2000) Pediatric cerebellar malformations: magnetic resonace imaging diagnostic merits and correlation with neurodevelopmental outcome. Egypt J Radiol Nucl Med 51:36

\section{Publisher's Note}

Springer Nature remains neutral with regard to jurisdictional claims in published maps and institutional affiliations. 